\title{
Genetic and clinical analysis in 10 Spanish patients with multiple endocrine neoplasia type 1
}

\author{
Aránzazu Cebrián ${ }^{1}$, Jose Luis Herrera-Pombo ${ }^{2}$, Juan José Díez ${ }^{3}$, Olga Sánchez-Vilar², \\ Jose Ignacio Lara ${ }^{2}$, Clotilde Vázquez ${ }^{4}$, Antonio Picó ${ }^{5}$, Ana Osorio ${ }^{1}$, \\ Beatriz Martínez-Delgado ${ }^{1}$, Javier Benítez ${ }^{1}$ and Mercedes Robledo ${ }^{1}$ \\ ${ }^{1}$ Department of Genetics and ${ }^{2}$ Department of Endocrinology, Fundación Jiménez Díaz, Madrid, Spain \\ ${ }^{3}$ Department of Endocrinology, La Paz Hospital, Madrid, Spain \\ ${ }^{4}$ Department of Endocrinology, Severo Ocho Hospital, Madrid, Spain \\ ${ }^{5}$ Department of Endocrinology and Nutrition, Universitary General Hospital, Alicante, Spain
}

\begin{abstract}
Multiple endocrine neoplasia type 1 (MEN1) is characterised by the combination of tumours of the parathyroid, endocrine pancreas and anterior pituitary glands. In 1988 the MEN 1 gene was mapped to chromosome 11q13 and it was cloned in 1997. This gene contains 10 exons and extends across $9 \mathrm{~Kb}$ of genomic DNA; it encodes for a product of 610 amino acid named menin whose function is unknown. We have studied 10 unrelated MEN 1 kindreds by a complete sequencing analysis of the entire gene; mutations were identified in nine of them: five deletions, one insertion, two nonsense mutation and a complex alteration consisting of a deletion and an insertion that can be explained by a hairpin loop model. Two of the mutations have been previously described; the other seven were novel, and they were scattered throughout the coding sequence of the gene. As in previous series, no correlation was found between phenotype and genotype.
\end{abstract}

Keywords: MEN 1 gene; tumor suppressor gene; germline mutations; MEN 1 kindreds; hairpin loop; truncated protein

\section{Introduction}

Multiple endocrine neoplasia type 1 (MEN1) is characterised by the combination of tumours of the parathyroid, endocrine pancreas and anterior pituitary glands. Over $90 \%$ of affected subjects present with hyperparathyroidism, HPT (hyperplasia or parathyroid tumours) by the age of 40, often involving all four

Correspondence: Mercedes Robledo, Department of Genetics, Fundación Jiménez Díaz, Avda Reyes Católicos 2, 28040 Madrid, Spain. Tel: 34 91 5449223; Fax: 3491 5448735; E-mail: mrobledo@uni.fjd.es

Received 13 July 1998; revised 12 January 1999; accepted 4 February 1999 parathyroid glands. ${ }^{1}$ In many cases, the clinical spectrum includes more complex lesions, such as neuroendocrine tumours (carcinoids), secreting or nonsecreting adrenal tumours, paraganglioma, thyroid adenoma and lipoma., ${ }^{2,3}$ MEN 1 is known to have very high penetrance, of about $98.8 \%$ by the age of $53 .{ }^{1}$ In 1988 the MEN 1 gene was mapped to chromosome 11 q13 by linkage analysis and deletion studies in tumoral DNA, ${ }^{4}$ and it was identified in 1997; this gene contains 10 exons (with the first exon untranslated) and extends across $9 \mathrm{~Kb}$. It encodes for a product of 610 amino acid named menin, ${ }^{5}$ that has been recently identified as a nuclear protein ${ }^{6}$ whose function is 
unknown. So far more than 70 different germ-line mutations have been described in selected MEN1 kindreds. These mutations are most commonly either nonsense mutations or small deletions, and they are scattered throughout the full length of the coding sequence of the gene. ${ }^{5-10}$ It has not been possible to establish a genotype-phenotype correlation. Moreover, loss of heterozygosity on 11q13, together with somatic mutations in the MEN 1 gene have been detected in approximately $20-27 \%$ of non-MEN 1 tumours of the parathyroid and the endocrine pancreas, ${ }^{11,12}$ confirming the role of $M E N 1$ as a tumour suppressor gene.

We report on a genetic analysis of 10 unrelated MEN 1 probands. All except one presented with germline mutations of which seven have not been previously described.

\section{Materials and Methods}

The families were studied in the Departments of Endocrinology of the four participating centres; Fundación Jiménez Díaz, La Paz and Severo Ochoa Hospitals (Madrid) and Universitary General Hospital (Alicante). MEN1 families were selected following the criteria previously described. ${ }^{13}$

Diagnosis of primary hyperparathyroidism was established by the simultaneous presence of elevated ionised or albuminadjusted serum calcium concentrations and intact parathyroid hormone levels at least twice. Histologic study after surgery defined adenoma or hyperplasia.

Pituitary adenomas were diagnosed by the presence of a mass lesion on high-resolution CT scanning or MR imaging of the sella turcica. Baseline hormonal concentrations and dynamic tests of pituitary reserve were employed to diagnose hormone-secreting tumours. Histopathology and immunohistochemistry analysis after surgical explorations of the pituitary fossa confirmed the presence of an adenoma or hyperplasia.

Pancreatic islet cell tumours were suspected on the evidence of clinical manifestations and elevated serum concentrations of pancreatic hormones (gastrin, insulin, glucagon, VIP, pancreatic polypeptide). Histologic study confirmed the presence of hyperplasia or neoplasia.

Informed consent was obtained from all patients. DNA was obtained from leukocyte of affected patients and their relatives using standard procedures. Subsequent PCR amplifications were performed in $20 \mu \mathrm{l}$ of a mixture containing $1 \times$ PCR buffer (Boehringer Mannheim, Germany), $200 \mu \mathrm{M}$ dNTP, 10 pmol of each primer flanking exons 2-10 of the MEN1 gene, ${ }^{7} 100-200 \mathrm{ng}$ of genomic DNA and $1 \mathrm{U}$ Taq polymerase (Boehringer Mannheim). PCR conditions were 10 cycles of $30 \mathrm{~s}$ at $94^{\circ} \mathrm{C}, 30 \mathrm{~s}$ at $68^{\circ} \mathrm{C}$ and $30 \mathrm{~s}$ at $72^{\circ} \mathrm{C}$, followed by 20 cycles of $30 \mathrm{~s}$ at $94^{\circ} \mathrm{C}, 30 \mathrm{~s}$ at $62^{\circ} \mathrm{C}$ or $63^{\circ} \mathrm{C}$ and $1 \mathrm{~min}$ at $72^{\circ} \mathrm{C}$ and with a $5 \mathrm{~min}$ final extension at $72^{\circ} \mathrm{C}$. The PCR products were purified by columns (Wizard PCR prep, Promega, Madison, WI) and bidirectionally sequenced with Drhodamine terminator cycle sequencing Kit (Applied Biosystems) according to the manufacturer's instructions. The PCR products were cloned to verify the mutant sequences in which an insertion and/or a deletion were observed. The pBluescript II K S ( + ) (Stratagene) was digested with EcoRV. The different PCR fragments were blunted with Klenow polymerase, and ligated to the digested plasmid. The positive colonies were bidirectionally sequenced with Drhodamine terminator cycle sequencing Kit (Applied Biosystems) using $\mathrm{T} 3$ and $\mathrm{T} 7$ primers.

The nonsense mutations were confirmed by restriction digest analysis: Q209X destroys a restriction site for Hae III enzyme and W341X generates a new restriction site for Dde I enzyme. Six polymorphic markers (D11S4076, D11S1765, AFMA350, D11S4205, D11S1883, D11S913) were used to establish the haplotype in the family with an apparently de novo case.

\section{Results}

A germline mutation was detected in $9 / 10$ studied probandi (Table 1) and all generated a truncated MEN 1 protein. Two of them had been previously described, a deletion of $2 \mathrm{bp}\left(2556 \mathrm{del}\right.$ AT (codon 90)) ${ }^{8}$ and a deletion of $1 \mathrm{bp}(7770 \mathrm{delC}(\operatorname{codon} 515)),{ }^{9}$ and the

Table 1 Clinical features and location of the detected mutations in the 10 patients studied

\begin{tabular}{|c|c|c|c|c|c|c|c|}
\hline $\begin{array}{l}\text { Patient } \\
\text { (Family) }\end{array}$ & $H P T$ & Pituitary tumours & Pancreatic tumours & Other findings & Mutation $^{\mathrm{a}}$ & Codon & Exon \\
\hline 1 & 6 & $2(\mathrm{P}, \mathrm{P})$ & $2(\mathrm{G}, \mathrm{G})$ & None & 2416delCGT & 43 & 2 \\
\hline 2 & 1 & $1(\mathrm{P})$ & $1(\mathrm{I})$ & None & 2530insC & 82 & 2 \\
\hline 3 & 9 & $3(\mathrm{P}, \mathrm{P}, \mathrm{P})$ & None & None & 2535del TGTC & 83 & 2 \\
\hline 4 & 7 & None & $4(\mathrm{~N}, \mathrm{G}, \mathrm{G}, \mathrm{G})$ & A & 2556delAT & 90 & 2 \\
\hline 5 & 2 & $2(\mathrm{P}, \mathrm{S})$ & None & None & Gln $\rightarrow$ Term & 209 & 3 \\
\hline 6 & 2 & $1(\mathrm{P})$ & None & A & 4799delCATT/ins9bp & 246 & 4 \\
\hline 7 & 2 & None & $1(\mathrm{I})$ & None & $\operatorname{Trp} \rightarrow$ Term & 341 & 7 \\
\hline 8 & 4 & $2(\mathrm{P})$ & $1(\mathrm{I})$ & A & 7736del25bp & 503 & 10 \\
\hline 9 & 6 & $2(\mathrm{P}, \mathrm{Co})$ & 5 (G, I, Ppoma, Ppoma, Ppoma) & None & 7770delC & 514 & 10 \\
\hline 10 & 3 & $1(\mathrm{~S})$ & None & None & None & - & - \\
\hline
\end{tabular}

aAll mutations introduce codon STOP; HPT: hyperparathyroidism; I: insulinoma; N: non-functional tumour; P: prolactinoma; G: gastrinoma; Ppoma: tumour secreting pancreatic polypeptide; S: somatotrophinoma; Co: corticotrophinoma; A: adrenal cortical tumour. 
other seven were novel mutations, a 2416delCGT (codon 43), 2530insC (codon 82), a 2535delTGTC (codon 83), two nonsense mutations (Q209X and W341X), a 7736del25bp (codon 503) and a complex alteration consisting of a deletion and an insertion 4799delCATT/insAGCGAGTCG (codon 246) that was confirmed by cloning (Figure 1). The rest of mutations were also confirmed by different methods, either by cloning or by the use of restriction enzymes (data not shown).

No significant phenotypic differences were found between the nine MEN 1 families with mutations and the MEN 1 family without mutation.

One patient (case 2) presented an apparently de novo mutation (2530insC) because no other member of the family had clinical manifestations that suggested the presence of the disease. To confirm this finding we analysed other relatives; there was available DNA from the patient's mother and from his brother, but not from his father, who had died at age 44 because of other

A
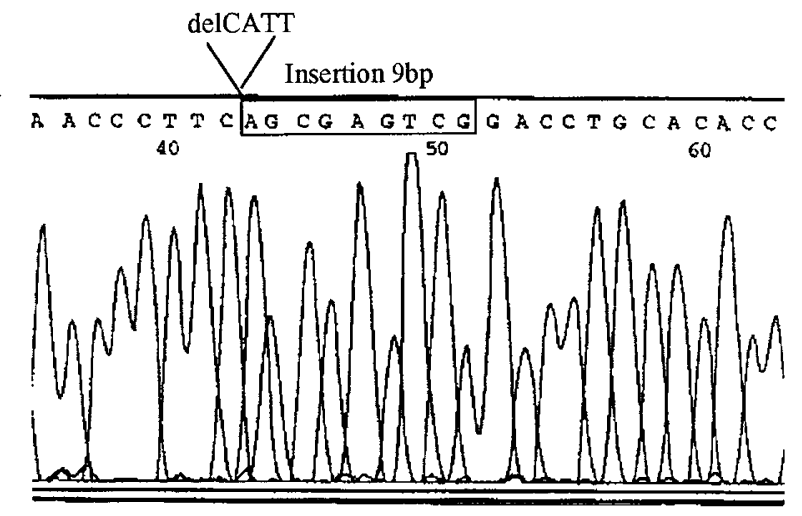

B

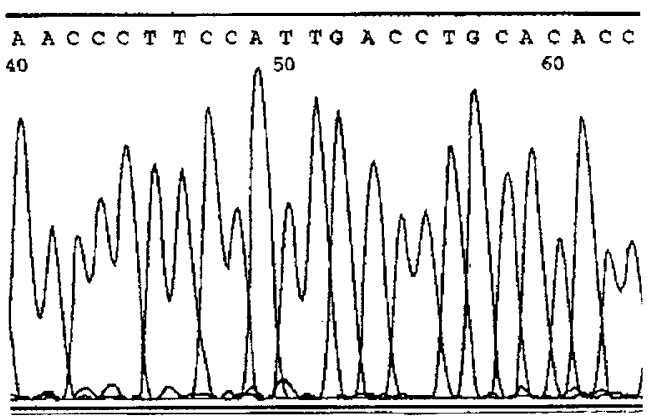

Figure 1 Sequence analysis in family 6 of the MEN 1 gene by cloning. A Mutated allele in exon 4, the deleted bases are indicated by an arrow, and insertion of nine bases is boxed; $\mathbf{B}$ normal allele. causes not related to the disease. The sequence analysis confirmed that none of them was a mutation carrier. We performed a familiar haplotype analysis using different microsatellite markers (D11S4076, D11S1765, AFMA350, D11S4205, D11S1883 and D11S913) trying to ascertain the character (hereditary or de novo) of the mutation. This analysis showed that the patient and his brother did not share the same paternal chromosome.

\section{Discussion}

We have studied 10 unrelated MEN1 patients: nine with a clear familial history and one apparently de novo case. In all cases except one, a germline mutation was detected, supporting the idea that mutations in the MEN 1 gene are involved in approximately $90 \%$ of families with this disease. ${ }^{9}$

Seven of these alterations were new mutations, located in exon 2 (2416delCGT, 2535delTGTC, 2530insC), exon 3 (Q209X), exon 4 (4799delCATT/ ins9bp), exon 7 (W341X) and exon 10 (7736del25bp), and two had been previously described (2556delAT, 7770delC) ${ }^{8,9}$ Four were deletions, one was an insertion and two were nonsense mutations; all generated a premature truncation of the MEN1 protein consistent with its putative role as a tumour suppressor gene., ${ }^{4,13}$

It is worth noting that $40 \%(4 / 10)$ of our cases exhibited a mutation in exon 2 which could suggest the existence of a region in this exon more frequently involved in the occurrence of mutations than has been previously reported. ${ }^{8}$

Two of the families (8 and 9), presented a mutation in exon 10. Both showed a similar phenotype to the other eight pedigrees, with an age of onset of 50 and 18 years respectively. These data support the hypothesis that the loss of the C-terminal portion of the protein is sufficient for functional inactivation. ${ }^{10}$ One of these mutations (7770delC) is located at the poly $(\mathrm{C})$ tract of codons 514-516, and has been recently defined as a possible hot spot in the MEN 1 gene. ${ }^{8}$

The MEN 1 gene appears to contain DNA sequences that could be susceptible to deletional and insertional events. In this sense, several mutations occur in regions of DNA that could be prone to DNA polymerase slippage during DNA replication due to short DNA sequence repeat motifs. ${ }^{14}$ The existence of CT and CA dinucleotide repeats has been observed flanking the $4 \mathrm{bp}$ deletions in the vicinity of the codons $83 / 84$ in exon 2 , and codons $210 / 211$ in exon 3 respectively, consistent with a replication slippage model. A similar 
event could have happened in the mutation located in exon 4. The complex alteration (del 4 bp/ins 9bp) (Figure 1), located in exon 4, could be explained by the formation of a hairpin loop structure (Figure 2) as has been already described in other diseases. ${ }^{15}$ There are several inverted repeated sequences around the region in which the mutation is located. To generate this complex mutation, these inverted repeated sequences could have formed a hairpin loop structure during replication and both the deletion and the insertion could have occurred within this structure. In the proposed mechanism, the alteration occurred as the combination of two steps, first a $4 \mathrm{bp}$ deletion and then a $9 \mathrm{bp}$ insertion. The deletion from nt 4729 to nt 4732 (CATT) could be due to the formation of a small side loop structure, and the inserted sequence (AGCGAGTCG) would correspond to the inverted complementary sequence from nt 4814 to nt 4822 (CGACTCGCT). This sequence could generate a misincorporation of the novel nine bases when the slippage, mediated by the other inverted sequences, occurred within this hairpin loop structure. This model could demonstrate that the dinucleotide repeats surrounding some of the insertions or deletions found in the MEN 1 gene are involved in the appearance of these mutational events, as has been suggested by other authors. $^{8,14}$

In family 2 only the proband showed clinical symptoms of MEN1 and carried a mutation in exon 2. By means of sequencing and haplotype analysis we could not confirm with $100 \%$ reliability that this was a de novo mutation, because the proband and his healthy brother had different paternal chromosomes. However, the absence of the mutation in his mother and the absence of clinical symptoms in his father (death at 44 years) support this hypothesis. On the other hand, $10 \%$ of the families described in the literature have de novo mutations, which has great clinical relevance. The appearance of MEN1 tumours in patients without a familial history does not obviate the risk of developing tumours within the siblings. In these cases it is necessary to have a clinical and biochemical follow-up after the carrier status has been determined.

Finally, we cannot disregard the fact that family 10, in which a mutation in the MEN 1 gene was not found, does not present an alteration in other regions such as the promotor region. In any case, we think that the sequencing of the entire sequence of the $M E N 1$ gene
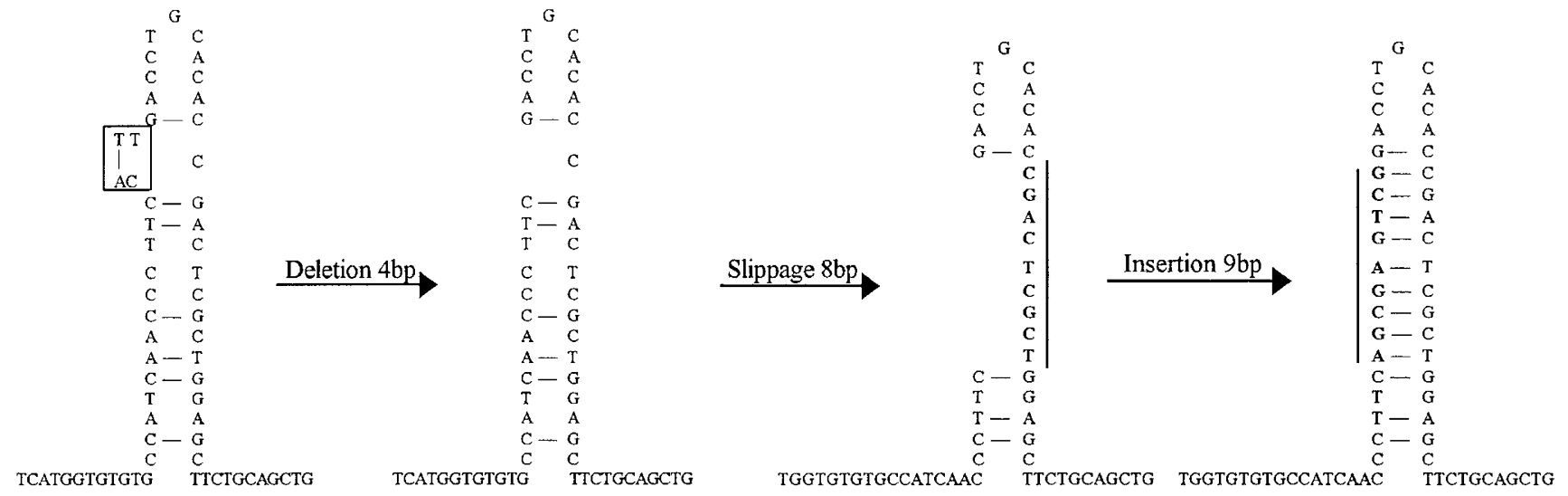

\section{Insertion

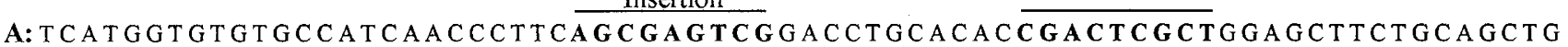

B: TCATGGTGTGTGCCATCAACCCTTCCATTGACCTGCACACCGACTCGCTGGAGCTTCTGCAGCTGCAGCAG

Figure 2 Proposed mechanism for the generation of the mutation in exon 4. The hairpin loop structure formation would be mediated by inverted repeated sequences. The first step is a four base deletion within the hairpin loop structure. Secondly there is an eight base slippage, and finally an insertion of nine bases occurs. The deleted bases are boxed and the insertion is the result of a replication of the nine bases indicated by a line. $\mathbf{A}$ alterated sequence as the result of the deletion/insertion mutational event; $\mathbf{B}$ normal sequence. 
as a screening method allows the mutations responsible for the disease to be characterised at a higher percentage than using other techniques such as the SSCP. ${ }^{16}$ In fact, SSCP does not detect all sequence changes, because the ability of each mutation to alter the conformation of the single strand depends on whether it occurs in a loop or in a long stable stem of the secondary structure, ${ }^{17}$ or on the size of the segment analysed. $^{18}$

In summary, we have found germline mutations in nine out of 10 patients with suspect MEN 1, supporting the theory that the MEN1 gene is responsible for this disease. The type of the mutations, their location and the clinical characteristics of this study, did not allow us to establish a genotype-phenotype correlation. More studies would be required to ascertain an association between mutations in concrete protein domains with certain phenotypes, as has been suggested for other tumour suppressor genes such as BRCA1 and 2 in familial breast cancer. ${ }^{19,20}$

\section{Acknowledgements}

This work has been partially supported by FIS 97/0339 and CAM 08.1/0024/1998. A Cebrián is a Fellow of Fondo de Investigaciones Sanitarias, and AOsorio is a Fellow of Conchita Rábago.

\section{References}

1 Trump D, Farren B, Wooding C et al: Clinical studies of multiple endocrine neoplasia type 1 (MEN1). $Q J$ Med 1996; 89: 653-669.

2 Lips CJM, Vasen HF, Lames CB: Multiple endocrine neoplasia (MEN) syndromes. Rev Oncol Hematol 1984; 2: 117.

3 Raue F, Zink A: Clinical features of multiple endocrine neoplasia type 1 and type 2. Horm Res 1992; 38: 31-35.

4 Larsson C, Skogseid B, Oberg K, Nakamura Y, Nordenskjöld M: Multiple endocrine neoplasia type 1 gene maps to chromosome 11 and is lost in insulinoma. Nature 1988; 332: $85-87$.

5 Chandrasekharappa SC, Guru SC, Manickam P et al: Positional cloning of the gene for multiple endocrine neoplasia - type 1. Science, Washington DC 1997; 276: 404-407.
6 Guru SC, Goldsmith PK, Burns AL et al: Menin, the product of the MEN 1 gene, is a nuclear protein. Proc Natl Acad Sci USA 1998; 95: 1630-1634.

7 European Consortion on MEN 1: Identification of the multiple endocrine neoplasia type 1 (MEN1) gene. Hum Mol Genet 1997; 6: 1177-1183.

8 Basset JHD, Forbes SA, Pannett AAJ et al: Characterization of mutations in patients with multiple endocrine neoplasia type 1. Am J Hum Genet 1998; 62: 232-244.

9 Agarwal SK, Kester MB, Debelenko LV et al: Germline mutations of the MEN1 gene in familial multiple endocrine neoplasia type 1 and related states. Hum Mol Genet 1997; 6: 1169-1175.

10 Debelenko LV, Brambilla E, Agarwal SK et al: Identification of MEN1 gene mutations in sporadic carcinoid tumors of the lung. Hum Mol Genet 1997; 6: 2285-2290.

11 Heppner C, Kester MB, Agarwal SK et al: Somatic mutations of the $M E N 1$ gene in parathyroid tumours. Nat Genet 1997; 16: 375-378.

12 Hessman O, Lindberg D, Skogseid B et al: Mutation of the multiple endocrine neoplasia type 1 gene in nonfamilial, malignant tumors of the endocrine pancreas. Cancer Res 1998; 58: 377-379.

13 Thakker RV, Bouloux P, Wooding D et al: Association of parathyroid tumors in multiple endocrine neoplasia type 1 with loss of alleles on chromosome 11. N Engl J Med 1989; 321: 218-224.

14 Agarwal SK, Debelenko LV, Kester MB et al: Analysis of recurrent germline mutations in the MEN 1 gene encountered in apparently unrelated families. Hum Mutat 1998; 12: $75-82$.

15 Yamakawa-Kobayashi K, Kobayashi T, Yanagi H, Shimakura Y, Satoh J, Hamaguchi H: A novel complex mutation in the LDL-receptor gene probably caused by the simultaneous occurrence of deletion and insertion in the same region. Hum Genet 1994; 93: 625-628.

16 Orita M, Suzuki Y, Sekiya T, Hayashi K: Rapid and sensitive detection of point mutations and DNA polymorphisms using the polymerase chain reaction. Genomics 1989; 5: 874-879.

17 White MB, Carvalho M, Derse D, O'Brien SJ, Dean M: Detecting single base substitutions as heteroduplex polymorphisms. Genomics 1992; 12: 301-306.

18 Sarkar G, Yoon H-S, Sommer SS: Dideoxy Fingerprinting (ddF): A rapid and efficient screen for the presence of mutations. Genomics 1992; 13: 441-443.

19 Gayther SA, Warren W, Mazoyer S et al: Germline mutations of the BRCA1 gene in breast and ovarian cancer families provide evidence for a genotype-phenotype correlation. Nat Genet 1995; 11: 428-433.

20 Gayther SA, Mangion J, Russel P et al: Variation of risks of breast and ovarian cancer associated with differen germline mutations of the BRCA2 gene. Nat Genet 1997; 15: $103-105$ 